\title{
Modeling wave-structure interactions by an immersed boundary method in a $\sigma$-coordinate model
}

\author{
Gangfeng $\mathrm{Ma}^{1}$, Arash A. Farahani ${ }^{1}$, James T. Kirby ${ }^{2}$ and Fengyan $\mathrm{Shi}^{2}$ \\ ${ }^{1}$ Department of Civil and Environmental Engineering, Old Dominion University, \\ Norfolk, VA, USA \\ ${ }^{2}$ Center for Applied Coastal Research, University of Delaware, Newark, DE, USA \\ Corresponding author: Tel: 1-757-683-4732; Email address: gma@odu.edu
}

\begin{abstract}
This paper studies wave-structure interactions using an immersed boundary (IB) method in a $\sigma$ coordinate non-hydrostatic wave model. In contrast to a fixed grid, the $\sigma$ grid follows the movement of the free surface, which may introduce numerical errors when applying the IB method. When the grid cells located inside the structure at the previous time step move into the flow domain at the current time step, a reconstruction of velocities at the cell center is conducted to accurately estimate the fluxes at the cell faces by enforcing the no-slip boundary condition at the structure boundaries. The model is validated against experimental data in three different problems, including solitary wave interactions with rectangular obstacles, periodic wave interaction with a submerged bar and non-breaking solitary wave interactions with multiple cylinders. These test cases deal with bottom-mounted, floating as well as emergent structures with different shapes. The agreements between simulations and measurements are generally good in terms of free surface ele-


vation, flow velocity, dynamic pressure and wave runup. It is shown that the IB method is a promising tool to simulate wave processes around structures and to predict wave/surge loads on near-coast structures.

Keywords: non-hydrostatic modeling, NHWAVE, immersed boundary method, wave-structure interactions

\section{1. Introduction}

2 Wave-structure interaction has become an increasingly important prob3 lem due to the need of accurately predicting wave/surge loads on near-coast

4 structures, which requires the understanding of flow characteristics as well as 5 wave processes such as wave scattering and reflection around the structures. 6 The boundary integral method (BIM) based on a potential flow theory (Sk7 ourup et al., 1992; Grilli et al., 1994; Koo and Kim, 2007) fail to reproduce 8 the vortices and turbulent flow structures, thus are not capable of accurately 9 predicting the pressure distributions and wave forces on structures, especially 10 with complex and irregular shapes. Recent trends are towards using a com11 putational fluid dynamics (CFD) model for this problem, for example, Hieu 12 and Tanimoto (2006); Shen and Chan (2008), Mo and Liu (2009); Finnegan 13 and Goggins (2012); Westphalen et al. (2012); Liu et al., (2013); Ha et al. 14 (2014). These studies used either a Navier-Stokes solver with a Volume-of15 Fluid (VOF) method or a Smoothed Particle Hydrodynamics (SPH) method 16 with the aid of proper turbulence closure to simulate turbulent flow character17 istics around the structures, which have been proved to be rather successful. 
The major issue of a CFD model is its expensive computational cost, which limits the usage of this type of model in a large-scale domain.

An alternative to the CFD model for wave-structure interaction is the non-hydrostatic wave model (Lin et al., 2002; Zijlema and Stelling, 2008; Ma et al., 2012), which has shown great potential for resolving wave dynamics, including wave breaking (Ma et al., 2012; Smit et al., 2013; Zijlema and Stelling, 2008; Derakhti et al., 2016b,c), nonlinear wave dynamics (Smit et al., 2014) as well as infragravity wave motions (Ma et al., 2014; Rijnsdorp et al., 2014). Compared to the CFD models, the non-hydrostatic wave models assume that the free surface is a single value function of horizontal coordinates, which dramatically simplifies the Navier-Stokes equations, resulting in an explicit equation for free surface elevation. This simplification would lead to much more efficient computation of free surface elevation as well as water depth. Most of the existing non-hydrostatic wave models are formulated in surface- and terrain-following $\sigma$ coordinate (Lin et al., 2002; Yun and Wu, 2004; Zijlema and Stelling, 2008; Ma et al., 2012), which essentially maps the vertical water column in the physical domain to a transformed space. With this coordinate transformation, a limitation on the computational domain has been imposed that neither water depth $h$ nor free surface $\eta$ can have discontinuity in order to avoid the local singularity (Lin, 2006). This limitation prohibits the application of the model to the problems with floating and emergent structures as well as bottom-mounted structures with vertical faces. To overcome this restriction, Lin (2006) proposed a 3D multiple-layer $\sigma$ 
coordinate model to simulate surface wave interactions with various types of structures including floating and emergent structures. His model performed well in the cases with a structure of simple geometry, i.e. rectangular block. However, this extension of the $\sigma$ coordinate model leads to a great deal of complexity in the resulting governing equations. In addition, it fails to deal with structures of irregular shapes and complex geometry. Its performance on wave interactions with multiple structures was not proved either.

In this paper, we propose to use an immersed boundary (IB) method (Fadlun et al., 2000) to simulate wave-structure interactions in a $\sigma$ coordinate non-hydrostatic model NHWAVE. Using this method, the solid structures are treated as virtual bodies and replaced by IB forces at their boundaries. The water depth and free surface at the locations of the structures are defined as the same as those without the structures so that their continuousness is not affected by the presence of structures of any types. The IB method was originally developed by Peskin (1982) to simulate cardiac mechanics and associated blood flow. Since then, numerous modifications and refinements have been proposed, and wide applications on fluid-structure interactions have been carried out (Mittal and Iaccarino, 2005). Recently, the IB method has also been introduced in VOF models to study wave-structure interactions (Shen and Chan, 2008; Shen et al., 2009; Ha et al., 2013). However, to our knowledge, no studies have ever been conducted to show the performance of IB method in a $\sigma$ coordinate wave model. Under the $\sigma$ coordinate, all the interior grid points are not fixed. They fluctuate up and down with the 
moving free surface in the physical domain. While the boundaries of the

65

66

rigid structures are fixed in space and time. As a result, the grid points around the structures may move into the fluid domain at one time and inside the structures at another time (Lin, 2006), which brings great challenges to implement the IB method using the direct forcing approach because the interpolation of the velocities may introduce large numerical errors.

The paper is organized as follows. The IB method and NHWAVE model formulations are introduced in section 2. Numerical simulations including solitary wave interactions with rectangular obstacles, periodic wave interactions with a submerged bar and non-breaking solitary wave interactions with multiple cylinders are presented in section 3. Finally, the conclusions are given in section 4.

\section{Formulations}

NHWAVE (Ma et al., 2012, Derakhti et al., 2016a) is a three-dimensional non-hydrostatic wave model that is capable of predicting instantaneous free surface and 3D flow fields. It is based on the assumption that the free surface is a single value function of horizontal coordinates. Fluid phenomena involving overturning of the free surface, such as plunging breaking waves, are not considered in this study. The governing equations of NHWAVE are the incompressible Navier-Stokes equations in well-balanced conservative form, formulated in time-dependent surface and terrain-following $\sigma$ coordi- 
85 nate, which is defined as

$$
t=t^{*} \quad x=x^{*} \quad y=y^{*} \quad \sigma=\frac{z^{*}+h}{D}
$$

86 where the total water depth $D(x, y, t)=h(x, y)+\eta(x, y, t)$.

87 With $\sigma$ coordinate transformation, the well-balanced mass and momen-

88 tum equations are given by

$$
\frac{\partial D}{\partial t}+\frac{\partial D u}{\partial x}+\frac{\partial D v}{\partial y}+\frac{\partial \omega}{\partial \sigma}=0
$$

89

$$
\frac{\partial \mathbf{U}}{\partial t}+\frac{\partial \mathbf{F}}{\partial x}+\frac{\partial \mathbf{G}}{\partial y}+\frac{\partial \mathbf{H}}{\partial \sigma}=\mathbf{S}_{h}+\mathbf{S}_{p}+\mathbf{S}_{\tau}
$$

90 where $\mathbf{U}=(D u, D v, D w)^{T}$ and $\omega$ is the vertical velocity in the $\sigma$ coordinate 91 image domain. The fluxes are

$$
\mathbf{F}=\left(\begin{array}{c}
D u u+\frac{1}{2} g \eta^{2}+g h \eta \\
D u v \\
D u w
\end{array}\right) \quad \mathbf{G}=\left(\begin{array}{c}
D u v \\
D v v+\frac{1}{2} g \eta^{2}+g h \eta \\
D v w
\end{array}\right) \quad \mathbf{H}=\left(\begin{array}{c}
u \omega \\
v \omega \\
w \omega
\end{array}\right)
$$

92 The first three source terms on the right hand side of equation (3) account 93 for the contributions from hydrostatic pressure, non-hydrostatic pressure and 
94
97

98

99

turbulent diffusion, given by

$$
\mathbf{S}_{h}=\left(\begin{array}{c}
g \eta \frac{\partial h}{\partial x} \\
g \eta \frac{\partial h}{\partial y} \\
0
\end{array}\right) \quad \mathbf{S}_{p}=\left(\begin{array}{c}
-\frac{D}{\rho}\left(\frac{\partial p}{\partial x}+\frac{\partial p}{\partial \sigma} \frac{\partial \sigma}{\partial x^{*}}\right) \\
-\frac{D}{\rho}\left(\frac{\partial p}{\partial y}+\frac{\partial p}{\partial \sigma} \frac{\partial \sigma}{\partial y^{*}}\right) \\
-\frac{1}{\rho} \frac{\partial p}{\partial \sigma}
\end{array}\right) \quad \mathbf{S}_{\tau}=\left(\begin{array}{c}
D S_{\tau_{x}} \\
D S_{\tau_{y}} \\
D S_{\tau_{z}}
\end{array}\right)
$$

${ }_{95}$ where $D S_{\tau_{x}}, D S_{\tau_{y}}, D S_{\tau_{z}}$ are diffusion terms. Written in a conservative form,

the diffusion terms are given by (Derakhti et al., 2016a)

$$
D S_{\tau i}=\frac{\partial 2\left(\nu+\nu_{t}\right) E_{i j}}{\partial x_{j}} \lambda_{j}+\frac{\partial 2 \sigma_{x_{j}^{*}}\left(\nu+\nu_{t}\right) E_{i j}}{\partial \sigma}
$$

where $\lambda_{j}=1-\delta_{3 j},(j=1,2,3), \nu$ is kinematic viscosity, $\nu_{t}$ is turbulent viscosity, $\sigma_{x_{j}^{*}}=\partial \sigma / \partial x_{j}^{*}$, and

$$
E_{i j}=\frac{1}{2}\left\{\frac{\partial U_{i}}{\partial x_{j}} \lambda_{j}+\frac{\partial U_{j}}{\partial x_{i}} \lambda_{i}+\frac{\partial}{\partial \sigma}\left(\sigma_{x_{j}^{*}} U_{i}+\sigma_{x_{i}^{*}} U_{j}\right)\right\}
$$

In order to simulate the turbulence flow around the structures, a simple Smagorinsky subgrid model is employed to calculate eddy viscosity in this paper. Because turbulence in the wave boundary layer is not the concern of the current study, this model is sufficient to capture very large eddies around structures. The turbulent viscosity is calculated by

$$
\nu_{t}=\left(C_{s} \Delta\right)^{2} \sqrt{S_{i j} S_{i j}}
$$

104 where $C_{s}$ is the Smagorinsky coefficient, which is taken as $0.1, \Delta$ is the filter 

$110=19$ (Cabot and Moin, 2000).

111

\subsection{Numerical schemes}

width, which is calculated as $\Delta=(\Delta x \Delta y \Delta \sigma D)^{1 / 3}$, and $S_{i j}=\frac{1}{2}\left(\frac{\partial u_{i}}{\partial x_{j}^{*}}+\frac{\partial u_{j}}{\partial x_{i}^{*}}\right)$ is the stress tensor. Instead of modeling every detail in the near-wall region, a wall function proposed by Cabot and Moin (2000) is adopted to approximate the eddy viscosity at the first cell adjacent to the wall:

$$
\frac{\nu_{t}}{\nu}=\kappa y_{w}^{+}\left(1-e^{-y_{w}^{+} / A}\right)^{2}
$$

where $y_{w}^{+}=y_{w} u_{\tau} / \nu$ is the distance to the wall in wall units, $\kappa=0.41$ and $A$

To solve the water depth $D$, we integrate the continuity equation (2) from $\sigma=0$ to 1 . By using the boundary conditions at the bottom and surface for $\omega$, we may obtain the equation for free surface movement.

$$
\frac{\partial D}{\partial t}+\frac{\partial}{\partial x}\left(D \int_{0}^{1} u d \sigma\right)+\frac{\partial}{\partial y}\left(D \int_{0}^{1} v d \sigma\right)=0
$$

The well-balanced continuity and momentum equations (2) and (3) are discretized by a combined finite-volume and finite-difference approach with a second-order Godunov-type scheme. Following the numerical framework of NHWAVE (Ma et al., 2012), the velocities are defined at the cell centers, while the pressure is defined at the vertically-facing cell faces in order to accurately prescribe zero pressure condition at the free surface. The twostage second-order nonlinear Strong Stability-Preserving (SSP) Runge-Kutta scheme (Gottlieb et al., 2001) is adopted for time stepping in order to obtain 


$$
\begin{aligned}
& \frac{\mathbf{U}^{*}-\mathbf{U}^{n}}{\Delta t}=-\left(\frac{\partial \mathbf{F}}{\partial x}+\frac{\partial \mathbf{G}}{\partial y}+\frac{\partial \mathbf{H}}{\partial \sigma}\right)^{n}+\mathbf{S}_{h}^{n} \\
& \frac{\mathbf{U}^{(1)}-\mathbf{U}^{*}}{\Delta t}=\mathbf{S}_{p}^{(1)}
\end{aligned}
$$

where $\mathbf{U}^{n}$ represents $\mathbf{U}$ value at time level $n, \mathbf{U}^{*}$ is the intermediate value in the two-step projection method, and $\mathbf{U}^{(1)}$ is the final first stage estimate.

At the second stage, the velocity field is updated to a second intermediate level using the same projection method, after which the Runge-Kutta algorithm is used to obtain a final value of the solution at the $n+1$ time level.

$$
\begin{aligned}
& \frac{\mathbf{U}^{*}-\mathbf{U}^{(1)}}{\Delta t}=-\left(\frac{\partial \mathbf{F}}{\partial x}+\frac{\partial \mathbf{G}}{\partial y}+\frac{\partial \mathbf{H}}{\partial \sigma}\right)^{(1)}+\mathbf{S}_{h}^{(1)} \\
& \frac{\mathbf{U}^{(2)}-\mathbf{U}^{*}}{\Delta t}=\mathbf{S}_{p}^{(2)} \\
& \mathbf{U}^{n+1}=\frac{1}{2} \mathbf{U}^{n}+\frac{1}{2} \mathbf{U}^{(2)}
\end{aligned}
$$


${ }_{137}\left(i+\frac{1}{2}\right)$ can be reconstructed by

$$
\mathbf{U}_{i+1 / 2}^{L}=\mathbf{U}_{i}+\frac{1}{2} \Delta x_{i} \Delta \mathbf{U}_{i}, \quad \mathbf{U}_{i+1 / 2}^{R}=\mathbf{U}_{i+1}-\frac{1}{2} \Delta x_{i+1} \Delta \mathbf{U}_{i+1}
$$

138 where $\Delta \mathbf{U}_{i}$ is the gradient of $\mathbf{U}$ at cell $\mathrm{i}$, which is estimated by

$$
\Delta \mathbf{U}_{i}=\operatorname{avg}\left(\frac{\mathbf{U}_{i+1}-\mathbf{U}_{i}}{x_{i+1}-x_{i}}, \frac{\mathbf{U}_{i}-\mathbf{U}_{i-1}}{x_{i}-x_{i-1}}\right)
$$

139 in which avg is a van Leer limiter, which is used to avoid spurious oscillations ${ }_{140}$ in the reconstruction data. The flux $\mathbf{F}\left(\mathbf{U}^{L}, \mathbf{U}^{R}\right)$ is estimated by solving a 141 local Riemann problem at each horizontal facing cell face.

$$
\mathbf{F}\left(\mathbf{U}^{L}, \mathbf{U}^{R}\right)= \begin{cases}\mathbf{F}\left(\mathbf{U}^{L}\right) & \text { if } \quad s_{L} \geq 0 \\ \mathbf{F}^{*}\left(\mathbf{U}^{L}, \mathbf{U}^{R}\right) & \text { if } \quad s_{L}<0<s_{R} \\ \mathbf{F}\left(\mathbf{U}^{R}\right) & \text { if } \quad s_{R} \leq 0\end{cases}
$$

142 where

$$
\mathbf{F}^{*}\left(\mathbf{U}^{L}, \mathbf{U}^{R}\right)=\frac{s_{R} \mathbf{F}\left(\mathbf{U}^{L}\right)-s_{L} \mathbf{F}\left(\mathbf{U}^{R}\right)+s_{L} s_{R}\left(\mathbf{U}^{R}-\mathbf{U}^{L}\right)}{s_{R}-s_{L}}
$$

${ }_{143}$ with wave speeds $s_{L}$ and $s_{R}$ defined by

$$
\begin{aligned}
& s_{L}=\min \left(u_{L}-c_{L}, u_{s}-c_{s}\right) \\
& s_{R}=\max \left(u_{R}+c_{R}, u_{s}+c_{s}\right)
\end{aligned}
$$


145 In the projection step (i.e. eqs. (10) and (12)), a Poisson equation can be

where $c_{L}=\sqrt{g D_{L}}, c_{R}=\sqrt{g D_{R}}, u_{s}$ and $c_{s}$ are estimated by

$$
\begin{aligned}
& u_{s}=\frac{1}{2}\left(u_{L}+u_{R}\right)+c_{L}-c_{R} \\
& c_{s}=\frac{c_{L}+c_{R}}{2}+\frac{u_{L}-u_{R}}{4}
\end{aligned}
$$

derived by applying the continuity equation (Ma et al., 2012). The Poisson equation can be discretized by finite difference method, resulting in a linear equation system with a coefficient matrix of 15 diagonal lines. The linear system is solved using the high performance preconditioner HYPRE software library.

\subsection{Immersed boundary method}

In the immersed boundary (IB) method, the structures are treated as virtual bodies and replaced by IB forces applied on their boundaries (Figure 1). To implement the IB method, an additional IB force is added to the projection step of the solver (Ha et al., 2014), i.e. equations (6) and (8).

$$
\frac{\mathbf{U}^{(k)}-\mathbf{U}^{*}}{\Delta t}=\mathbf{S}_{p}^{(k)}+\mathbf{f}_{I B F}^{(k)}
$$

where $k=1,2$ at the first and second stages, respectively. The IB force $\mathbf{f}_{I B F}$ is defined as a Dirac delta function, which has non-zero values only at the structure boundaries. An IB velocity $\hat{\mathbf{U}}$ is employed to enforce a no-slip boundary condition at the structure boundaries. Thus the IB forcing is given 
160 by

$$
\mathbf{f}_{I B F}^{(k)}=\frac{\hat{\mathbf{U}}^{(k)}-\mathbf{U}^{*}}{\Delta t}-\mathbf{S}_{p}^{(k)}
$$

The IB force is applied at the cell center in the fluid domain nearest the solid surface. To find the IB velocity at the cell center, an interpolation is usually required. In this study, the linear interpolation approach proposed by Fadlun et al. (2000) is employed. The details of the interpolation scheme can also be referred to Ha et al. (2014). As shown in Figure 1, the IB velocities at the center of the cells nearest the structure are obtained by linearly interpolating the velocities at the structure boundary and the neighboring cell.

In Figure 1, we see that the computational grids in the $\sigma$ coordinate follow the movement of the free surface. The cell center, where the velocities are defined, could be located inside the structure at one time and move into the flow domain at another time. This always happens in the cases with submerged and floating structures. If the cell center is within the structure, its velocities are assumed to be the same as the velocities of the structure. When the cells located inside the structure at the previous time step move into the flow domain at the current time step, reconstruction of the velocities at the previous time step is needed to accurately estimate the fluxes at the cell faces by enforcing the no-slip boundary condition at the structure boundaries. 


\section{Numerical simulations}

\subsection{Solitary wave propagation through rectangular obstacles}

The model is firstly applied to study solitary wave propagation through a bottom-mounted rectangular obstacle. Both laboratory measurement (Zhuang and Lee, 1996) and numerical simulation (Lin, 2006) showed that a strong vortex structure is formed behind the obstacle during the passage of the solitary wave and it persists for a very long time. It is extremely important to capture this vortex structure in order to predict the wave forces on the obstacle.

The model setup is shown in Figure 2(a). The computational domain is $5 \mathrm{~m}$ long, discretized by 2000 grid cells with a uniform grid size of 2.5 mm. 40 vertical layers are employed in order to well capture the vortical structure behind the obstacle. The water depth is constant with $h=0.228$ m. The incident solitary wave has a wave height of $0.069 \mathrm{~m}$. The rectangular obstacle has a height of $h / 2=0.114 \mathrm{~m}$ and a length of $L=0.381 \mathrm{~m}$. At the boundaries of the obstacle, the IB forcing is applied. The temporal variations of horizontal as well as vertical velocities are measured at two points behind the obstacle. Point 1 is located at $0.040 \mathrm{~m}$ above the bottom and 0.034 $\mathrm{m}$ downstream from the obstacle, and point 2 is at $0.017 \mathrm{~m}$ above point 1. To accurately simulate the evolution of the vortical structure, proper turbulence model must be included. In this study, a Smagorinsky Sub-Grid Scale turbulence model is employed.

Figure 2(b)-(d) show the simulated flow fields during the passage of the 
solitary wave near the obstacle. We can clearly see the development and growth of the vortical structure behind the obstacle. The vortex is developed when the wave crest reaches the rear boundary of the obstacle. As the solitary wave passes by, the vortex grows in both size and strength. The core of the vortex tends to move downward in the direction of wave propagation and rise upward towards the free surface. The IB method implemented in NHWAVE can well capture the effects of the obstacle on the flow fields under the solitary wave.

Figure 3 presents the model-data comparisons of time series of horizontal and vertical velocities at two measurement points. It can be seen that simulations agree very well with the measurements at both points. The vortical structure initiates at around $t \sqrt{g / h}=12.0$, accompanied by the increase of both horizontal and vertical velocities in magnitude. The horizontal velocities are negative, indicating the development of reverse flow behind the obstacle, which is accurately captured by the present model. Figure 3 also includes the simulated velocities with doubled grid cells. Clearly, the results with coarse and fine grids have minor difference, indicating that the current grid is sufficiently fine to capture the flow field around the obstacle.

To evaluate the model skill on simulating waves interacting with a floating object, a new simulation is conducted (Figure 4). The computational domain is $50 \mathrm{~m}$ long, discretized by 1000 grid cells with a uniform grid size of 0.05 m. 40 vertical layers are again used in the vertical direction to capture the complicated flow field around the floating object. The water depth is $1.0 \mathrm{~m}$. 
The incident solitary wave has a wave height of $0.1 \mathrm{~m}$. The floating object has a rectangular shape with $5 \mathrm{~m}$ long and $0.4 \mathrm{~m}$ high, located at $(x, y)=(20.0$, 0.4) $\mathrm{m}$. Due to the lack of experimental data, this problem is also simulated by a validated Navier-Stokes solver TRUCHAS (Ma et al., 2011; Derakati and Kirby, 2014), which uses a Volume-of-Fluid (VOF) approach to capture the solid-fluid as well as water-air interfaces. The results at three gauges from both models are compared in Figure 4. Clearly, the discrepancies are negligible, indicating that the current model is able to accurately simulate waves interacting with a floating body.

The model's capability of simulating 3D interactions between solitary waves and a block is demonstrated through reproducing the laboratory experiments of Lara et al. (2012). The experiment was performed in the University of Cantabria wave flume, which is $20.62 \mathrm{~m}$ long, $0.58 \mathrm{~m}$ wide and $0.8 \mathrm{~m}$ high. The impervious rectangular block has dimensions of $30 \mathrm{~cm}$ in the cross-flume direction and $24 \mathrm{~cm}$ in the along-flume direction as shown in Figure 5. Its closest face to the wavemaker was located $10.84 \mathrm{~m}$ away from the wavemaker mean position. The case with the highest wave height (14 $\mathrm{cm})$ is selected for numerical simulation. The still water depth is $0.45 \mathrm{~m}$. A series of $15 \mathrm{~s}$ is simulated, which includes the first impact of the wave with the structure and the first reflection that reaches the obstacle again. The computational is discretized by a uniform grid with $\Delta x=0.02 \mathrm{~m}$ and $\Delta y=$ $0.01 \mathrm{~m} .20$ vertical layers are employed in order to better capture the vortices generated around the obstacle. The simulation takes about 15 hours, which 
is much faster than that of a VOF simulation using OpenFOAM (Higuera et al., 2013).

Figure 6 and 7 compare the simulated and measured free surface elevations at 12 wave gauges. Generally, the agreements are excellent, except that small discrepancies can be found at gauge 5 and 6 , where the results may be affected by the big eddies around the obstacle generated during the passage of the solitary wave. The leading wave as well as the reflected wave from the end wall are both well captured by the model.

\subsection{Periodic wave over a submerged bar}

In this section, the model is used to examine the interactions between dispersive waves and a submerged obstacle, which has two slopes that cut through the computational cells. The experimental data collected by Beji and Battjes (1993) are employed to validate the model. The model setup is shown in Figure 8. The water depth is $0.4 \mathrm{~m}$ over the whole domain. The incident periodic waves have a period of $2.02 \mathrm{~s}$ and a wave height of $2.0 \mathrm{~cm}$. The submerged bar is simulated using the IB method. It has a height of $0.3 \mathrm{~m}$. The offshore slope of the bar is $1 / 20$ and the onshore slope is $1 / 10$. The computational domain is discretized by 1750 grid cells with a uniform grid size of $0.02 \mathrm{~m}$. 40 vertical layers are employed to capture the flow field around the bar.

Figure 9 shows the model-data comparisons of free surface elevations at six measurement gauges. Wave shoaling at station a and propagation over 
the bar at station $b$ are well simulated by the model. The bound higher harmonics generated by the nonlinear shoaling wave on the upward slope of the bar become free on the downward slope, resulting in irregular wave pattern at station c-f. The model generally predicts free surface evolution at these stations well, indicating that dispersion of the higher frequency components is well simulated. It is proved that interactions between dispersive waves and submerged obstacle are well captured by the current model.

\subsection{Non-breaking solitary wave interacting with a group of slender vertical cylinders}

In this section, we will examine the capability of the current model on simulating wave interactions with multiple objects. The experimental data collected by Mo and Liu (2009) are used to check the accuracy of the model. The experiments were conducted in the Tsunami Wave Basin at the O.H. Hinsdale Wave Research Laboratory at Oregon State University and examined the interactions between non-breaking solitary waves and a group of three vertical cylinders. The wave basin has an effective length of $48.8 \mathrm{~m}$, a width of $26.5 \mathrm{~m}$ and a depth of $2.1 \mathrm{~m}$. Three stainless steel circular cylinders with a diameter of $D=1.219$ m were instrumented and installed in the basin. The time series of free surface elevations were collected at 10 wave gauges. The velocities were measured by 5 Acoustic Doppler Velocimetries (ADVs). The details of the experimental setup is referred to Mo and Liu (2009). The model setup is shown in Figure 10. To reduce the computational cost, 
the domain is chosen to be $30 \mathrm{~m}$ long and $7 \mathrm{~m}$ wide, which covers only a half of the wave basin because of the symmetric arrangement of the locations for cylinders. The width of the domain is designed to be greater than $5 D$ to ensure that wave reflection from the lateral walls does not contaminate the results in the region of interest within the simulation time. The water depth is $0.75 \mathrm{~m}$. The incident solitary wave has a wave height of $H=0.3 \mathrm{~m}$. A 6 $m$ wide sponge layer is placed at the end of the domain to absorb the wave energy. The placement of the wave gauges and ADVs are shown in Figure 7. The domain is discretized by $600 \times 140$ grid cells with the grid size of $\Delta x$ $=0.05 \mathrm{~m}$ and $\Delta y=0.05 \mathrm{~m} .10$ vertical layers are employed to capture the vertical structure of velocity field. A simple Smagorinsky Sub-Grid Scale model is used to simulate turbulent flow.

Figure 11 demonstrates the interactions between the solitary wave and three vertical cylinders. The wave reaches at the cylinders nearest the wavemaker around $t / \sqrt{h / g}=25.32$. The water level is the highest in the front of the cylinders due to the wave runup. After the wave passes by the first low of the cylinders at $t / \sqrt{h / g}=28.93$, the highest water level is found behind the cylinders due to wave focusing and wave runup at the farthest cylinder. At $t / \sqrt{h / g}=32.55$, the wave has completely propagated through the cylinder group. The water level behind the farthest cylinder is high purely due to focusing of the diffracted waves.

The comparisons of simulated and measured free surface elevations at wave gauges are presented in Figure 12, in which the free surface elevations 
are normalized by the incident wave height $H$. Excellent agreements are observed at most wave gauges. Small discrepancy is found at gauge 5, which is located at the rear end of the first two cylinders. More importantly, the secondary waves generated by scattering from the cylinders are well captured by the current model. These comparisons are even better than those of a VOF simulation by Mo and Liu (2009), in which noticeable differences appeared for the secondary scattered waves.

The comparisons of simulated and measured flow velocities at 5 ADVs are displayed in Figure 13. At ADV1, which is located between the first two cylinders nearest to the wavemaker, the comparisons of three components of velocity are all excellent. At ADV2 and ADV3, where the flow separation takes place, noticeable differences in the horizontal velocities are observed. Specifically, the streamwise velocity $u$ is slightly over predicted at ADV2 while it is underestimated at ADV3. The prediction of the flow field within the cylinder group probably requires a more sophisticated turbulence model. At ADV4 and ADV5, the simulations generally agree well with the measurements.

The predicted wave runup in the front of the farthest cylinder is examined in Figure 14. Excellent agreement between simulation and measurement is obtained, indicating that the current model can well predict the wave processes around the cylinders. The maximum wave runup is about 1.5 times larger than the incident wave height. The simulated and measured dynamic pressures at $0.1 \mathrm{~m}$ above the bed in the front of the farthest cylinder are 
also compared in Figure 14. The dynamic pressure is normalized by $\rho g H$, where $\mathrm{H}$ is the incident wave height. The peak dynamic pressure during wave runup on the cylinder is accurately predicted the model. However, the dynamics pressure is slightly underestimated during wave run-down. The model generally predicts well the wave runup and run-down processes on the cylinder.

\section{Conclusions}

This paper implemented an immersed boundary (IB) method in a $\sigma$ coordinate non-hydrostatic model NHWAVE to simulate wave-structure interactions. In contrast to a fixed grid, the $\sigma$ grid follows the movement of the free surface. The grid cell center, where the velocities are defined, is located inside the structure at one time and can move into the flow domain at another time. When this situation takes place, a reconstruction of the flow velocities at the previous time step is needed in order to accurately estimate the fluxes at the cell faces. The IB-NHWAVE model was utilized to study solitary wave interactions with rectangular obstacles, periodic wave interactions with a submerged bar and non-breaking solitary wave interactions with multiply cylinders. The simulations agreed well with the measurements in terms of free surface elevation, velocity, dynamic pressure as well as wave runup. The structures in these test cases have different shapes and their boundaries may cut through the grid cells. It has been shown that the IB-NHWAVE is a promising tool to predict wave/surge loads on near-coast structures. 


\section{Acknowledgments}

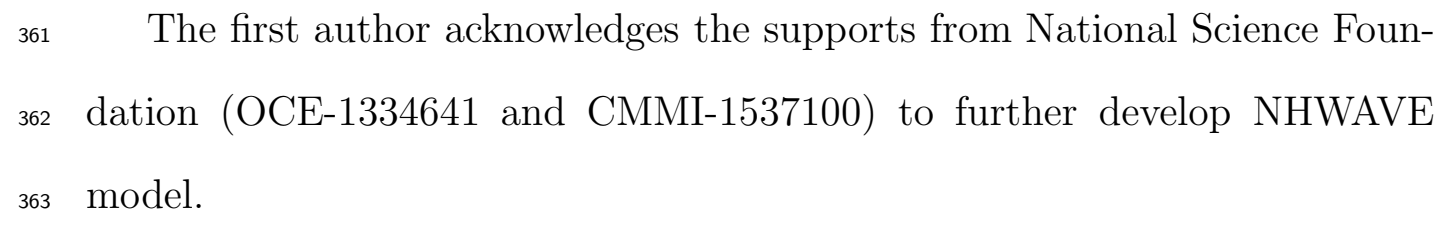




\section{References}

Beji S. and Battjes J.A., 1993, Experimental investigation of wave propagation over a bar, Coastal Eng., 19, 151162

Cabot W. and Moin P., 2000, Approximate wall boundary conditions in the large-eddy simulation of high Reynolds number flow, Flow Turb. Combust., 63, 269-291

Derakhti M. and Kirby J. T., 2014, Bubble entrainment and liquid-bubble interaction under unsteady breaking waves, Journal of Fluid Mechanics, 761, 464-506

Derakhti M., Kirby J. T., Shi F. and Ma G., 2016a, NHWAVE: Governing equations, exact boundary conditions and turbulence modeling, Ocean Modelling, under review

Derakhti M., Kirby J. T., Shi F. and Ma G., 2016b, Wave breaking in the surf zone and deep water in a non-hydrostatic RANS model. Part 1: Organized wave motions, Ocean Modelling, under review

Derakhti M., Kirby J. T., Shi F. and Ma G., 2016c, Wave breaking in the surf zone and deep water in a non-hydrostatic RANS model. Part 2: Breaking-induced velocity and turbulence, Ocean Modelling, under review

Fadlun E.A., Verzicco R., Orlandi P. and Mohd-Yusof J., 2000, Combined 
immersed-boundary finite-difference methods for three-dimensional complex flow simulations, Journal of Computational Physics, 161, 35-60

Finnegan W. and Goggins J., 2012, Numerical simulation of linear water waves and wave-structure interaction, Ocean Engineering, 43, 23-31

Gottlieb S., Shu C.-W. and Tadmor E., 2001, Strong stability-preserving high-order time discretization methods, SIAM Review, 43, 89-112

Grilli S.T., Losada M.A. and martin F., 1994, Characteristics of solitary wave breaking induced by breakwaters, Journal of Waterway, Port, Coastal and Ocean Engineering, 120, 74-92

Ha T., Shim J., Lin P. and Cho Y., 2014, Three-dimensional numerical simulation of solitary wave run-up using the IB method, Coastal Engineering, 84, 38-55

Hieu P.D. and Tanimoto K., 2006, Verification of a VOF-based two-phase flow model for wave breaking and wave-structure interactions, Ocean Engineering, 33, 1565-1588

Higuera P., Lara J.L. and Losada I.J., 2013, Simulating coastal engineering processes with OpenFOAM, Coastal Engineering, 71, 119-134

Koo W.C. and Kim M.H., 2007, Fully nonlinear wave-body interactions with surface-piercing bodies, Ocean Engineering, 34, 1000-1012 
Lara J.L., del Jesus M. and Losada I.J., 2012, Three-dimensional interaction of waves and porous coastal structures, Part II: Experimental validation, Coastal Engineering, 64, 26-46

Lin P. and Li C.W., 2002, A $\sigma$-coordinate three-dimensional numerical model for surface wave propagation, International Journal of Numerical Methods in Fluids, 38, 1045-1068

Lin P., 2006, A multiple-layer $\sigma$-coordinate model for simulation of wavestructure interaction, Computers \& Fluids, 35, 147-167

Liu X., Xu H., Shao S. and Lin P., 2013, An improved incompressible SPH model for simulation of wave-structure interaction, Computers \& Flu$i d s, 71,113-123$

Ma G., Shi F. and Kirby J. T., 2011, A polydisperse two-fluid model for surf zone bubble simulation, Journal of Geophysical Research, 116, C05010, doi:10.1029/2010JC006667

Ma G., Shi F. and Kirby J.T., 2012, Shock-capturing non-hydrostatic model for fully dispersive surface wave processes, Ocean Modelling, 43-44, 2235

Ma G., Su S.-F., Liu S. and Chu J.-C., 2014, Numerical simulation of infra gravity waves in fringing reefs using a shock-capturing non-hydrostatic model, Ocean Engineering, 85, 54-64 
Mittal R. and Iaccarino G., 2005, Immersed boundary methods, Annu. Rev. Fluid Mech., 37, 239-261

Mo W. and Liu P. L.-F., 2009, Three dimensional numerical simulations for non-breaking solitary wave interacting with a group of slender vertical cylinders, Inter J. Nav. Archie. Oc. Engine., 1, 20-28

Peskin C.S., 1982, The fluid dynamics of heart valves: experimental, theoretical and computational methods, Annu. Rev. Fluid Mech., 14, $235-259$

Rijnsdorp D.P., Smit P.B. and Zijlema M., 2014, Non-hydrostatic modelling of infragravity waves under laboratory conditions, Coastal Engineering, $85,30-42$

Skourup J., Sterndorff M.J. and Hansen E.A., 1992, Numerical modeling of wave-structure interaction by a three-dimensional non-linear boundary element method: A step towards the numerical wave tank, Ocean Engng., 19, 437-460

Shen L. and Chan E.-S., 2008, Numerical simulation of fluid-structure interaction using a combined volume of fluid and immersed boundary method, Ocean Engineering, 35, 939-952

Shen L., Chan E.-S. and Lin P., 2009, Calculation of hydrodynamic forces acting on a submerged moving object using immersed boundary method, Computer \& Fluids, 38, 691-702 
Smit P., Zijlema M. and Stelling G.S., 2013, Depth-induced wave breaking in a non-hydrostatic, near-shore wave model, Coastal Engineering, 76, $1-16$

Smit P., Janssen T., Holthuijsen L. and Smith J., 2014, Non-hydrostatic modeling of surf zone wave dynamics, Coastal Engineering, 83, 36-48

Westphalen J., Greaves D.M., Williams C.J.K., Hunt-Raby A.C. and Zang J., 2012, Focused waves and wave-structure interaction in a numerical wave tank, Ocean Engineering, 45, 9-21

Yuan H. and Wu C.H., 2004, A two-dimensional vertical non-hydrostatic $\sigma$ model with an implicit method for free-surface flows, International Journal For Numerical Methods In Fluids, 44, 811-835

Zhuang F. and Lee J.J., 1996, A viscous rotational model for wave overtopping over marine structure, Proc. 25th Int. Conf. Coast. Eng., ASCE, $2178-2191$

Zijlema M. and Stelling G.S., 2008, Efficient computation of surf zone waves using the nonlinear shallow water equations with non-hydrostatic pressure, Coastal Engineering, 55, 780-790 
Figure 1: Illustration of IB method and linear interpolations of IB velocities in a $\sigma$ coordinate model. The $\sigma$ grid temporally varies with the movement of the free surface. The dashed ellipse indicates the boundary of the structure, where the IB forces are applied. 
Figure 2: (a) Model setup of a solitary wave past a submerged obstacle and the simulated vortical structures behind the obstacle at (b) $t \sqrt{g / h}=12.64$; (c) $t \sqrt{g / h}=13.95$ and (d) $t \sqrt{g / h}=15.27$. The two circles behind the obstacle indicate the locations of measurement points 1 (lower) and 2 (upper). 

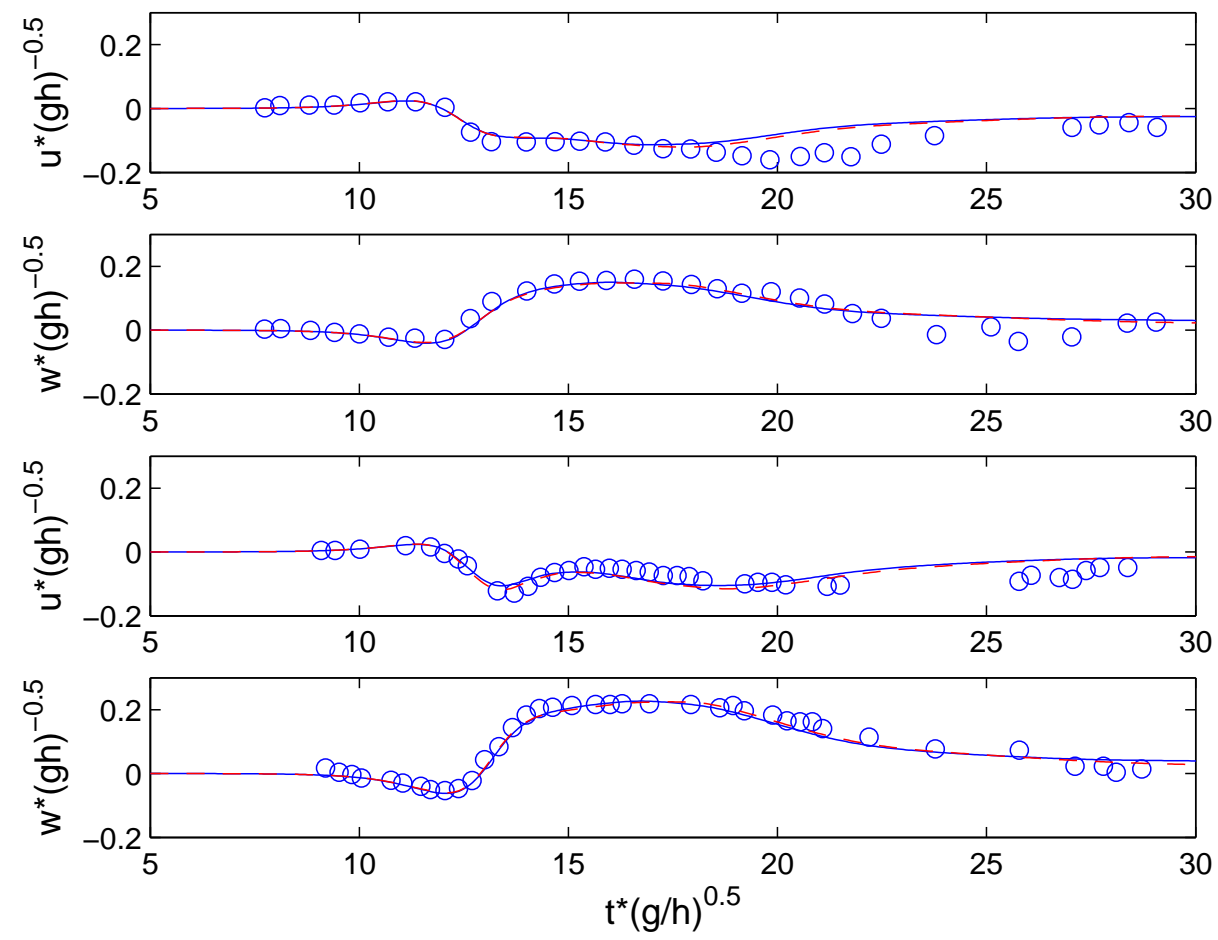

Figure 3: Model-data comparisons of the time series of horizontal and vertical velocities at two points behind the obstacle. Upper two panels show the results at point 1 and lower two panels show the results at point 2. Circles: experimental data; Solid lines: simulation results with $2000 \times 40$ grid cells; Dashed lines: simulation results with $4000 \times 40$ grid cells. 
Figure 4: Model setup of a solitary wave propagation through a floating object and the comparisons of simulated surface elevationset (a) gauge 1, (b) gauge 2 and (c) gauge 3 using the present model (solid lines) and a VOF model (dashed lines). 


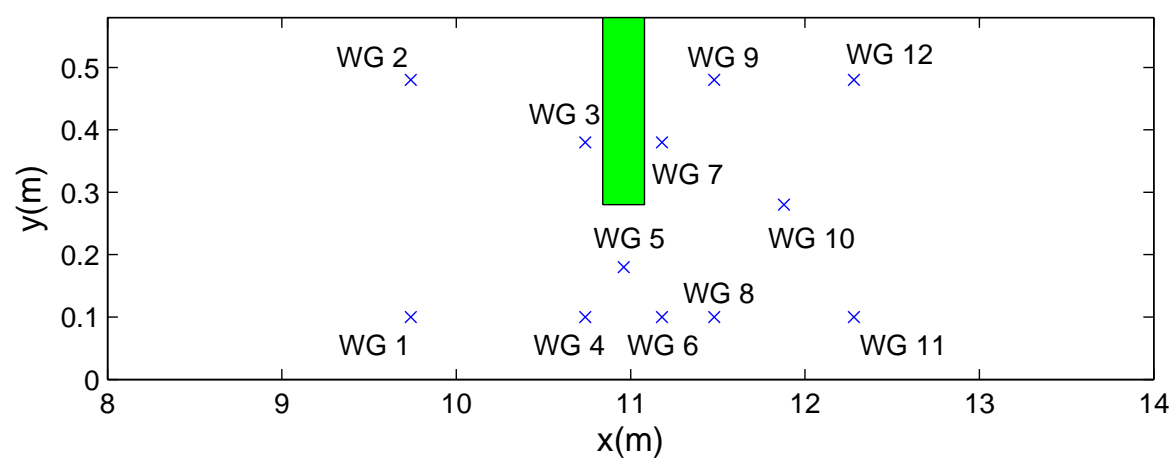

Figure 5: Computational domain for 3D interactions between a solitary wave and a block and the locations of the wave gauges. Solid lines: simulations; dashed lines: measurements 
Figure 6: Comparisons of simulated and measured free surface elevations at the first six wave gauges. Solid lines: simulations; dashed lines: measurements 
Figure 7: Comparisons of simulated and measured free surface elevations at the last six wave gauges. 


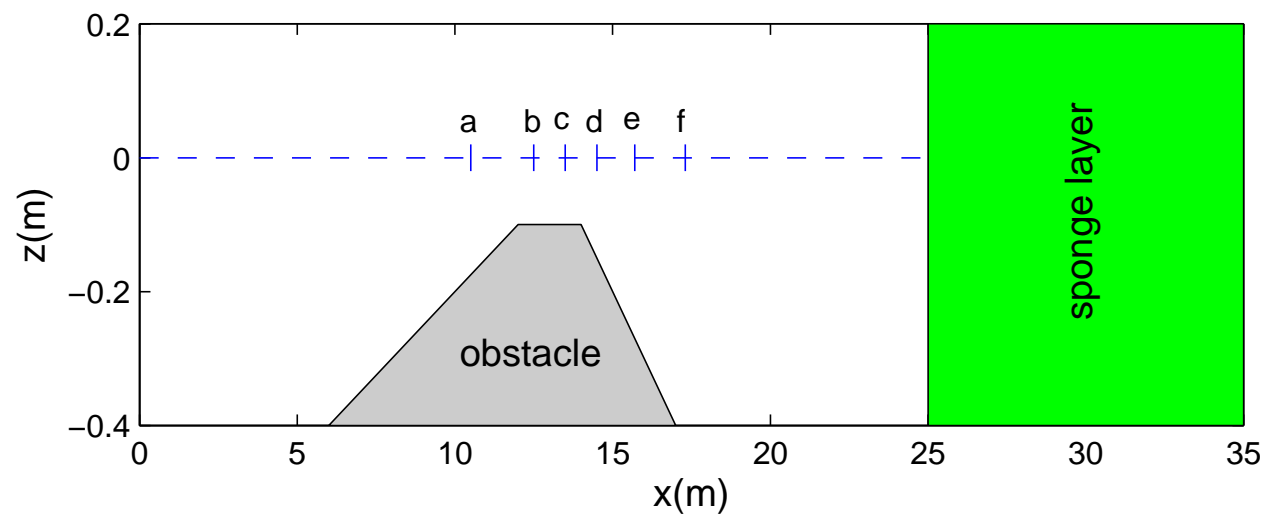

Figure 8: Model setup for the periodic waves interacting with a submerged bar. The locations of six wave gauges are located at (a) $x=10.5 \mathrm{~m}$; (b) $x=12.5 \mathrm{~m}$; (c) $x=13.5$ $\mathrm{m}$; (d) $x=14.5 \mathrm{~m}$; (e) $x=15.7 \mathrm{~m}$ and (f) $x=17.3 \mathrm{~m}$. A $10 \mathrm{~m}$ wide sponge layer is placed at the right end of the domain to absorb wave energy. 

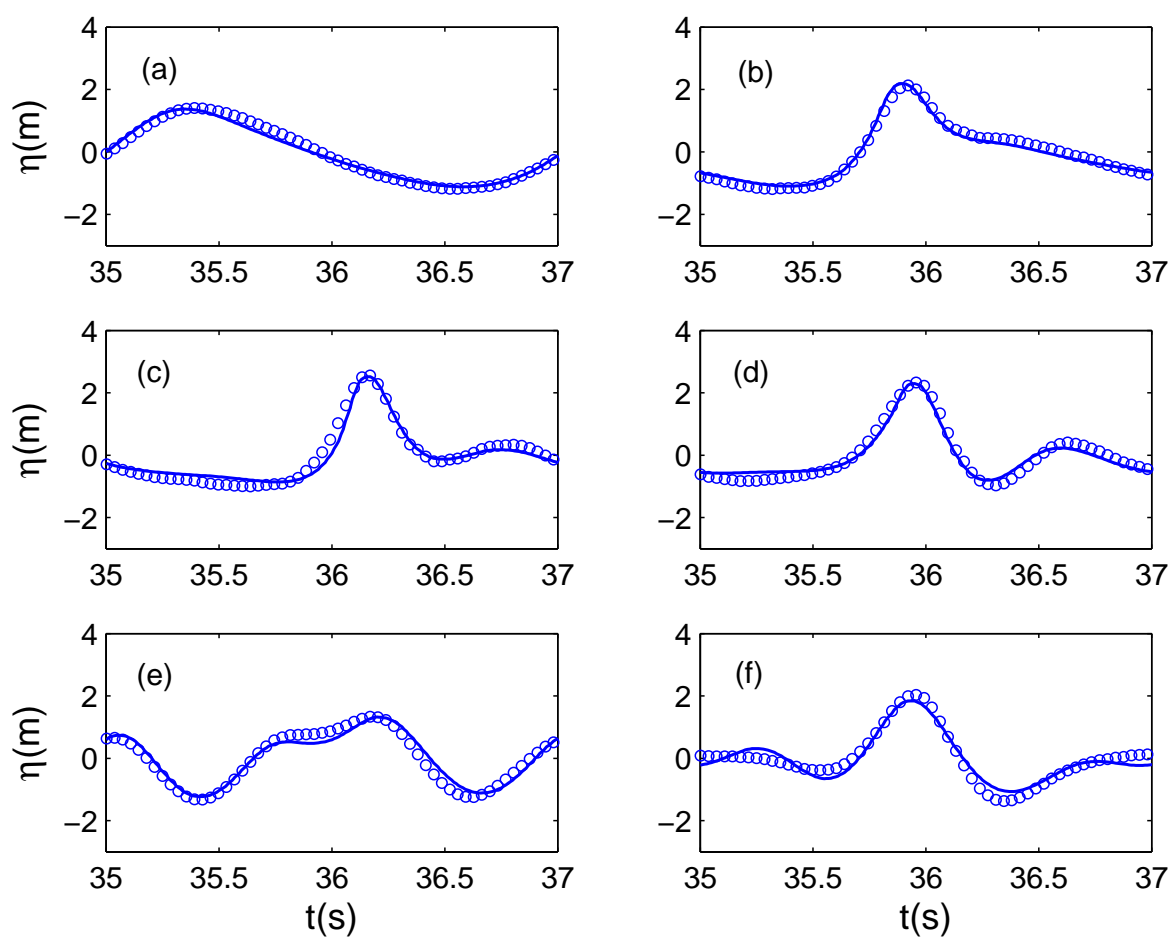

Figure 9: Comparisons of free surface elevations between simulations (solid lines) and measurements (circles) at (a) $x=10.5 \mathrm{~m}$; (b) $x=12.5 \mathrm{~m}$; (c) $x=13.5 \mathrm{~m}$; (d) $x=14.5$ $\mathrm{m} ;$ (e) $x=15.7 \mathrm{~m}$ and (f) $x=17.3 \mathrm{~m}$. 
Figure 10: Model setup for a solitary wave interacting with three slender vertical cylinders. The computational domain covers the region with $y>0$. Three cylinders are located at $(\mathrm{x}, \mathrm{y})=(21.085,0.0),(18.6595,1.219)$ and $(18.6595,-1.219) \mathrm{m}$, respectively. The diameter of the cylinder is $D=1.219 \mathrm{~m}$. The dots indicate the locations of 10 wave gauges deployed to measure the free surface elevations. The triangles indicate the locations of ADV measurements. A 6 m wide sponge layer is placed at the right end of the domain to absorb wave energy. 
Figure 11: Solitary wave interacting with three slender vertical cylinders at (a) $t / \sqrt{h / g}$ $=25.32 ;(\mathrm{b})=t / \sqrt{h / g}=28.93$ and $(\mathrm{c}) t / \sqrt{h / g}=32.55$.

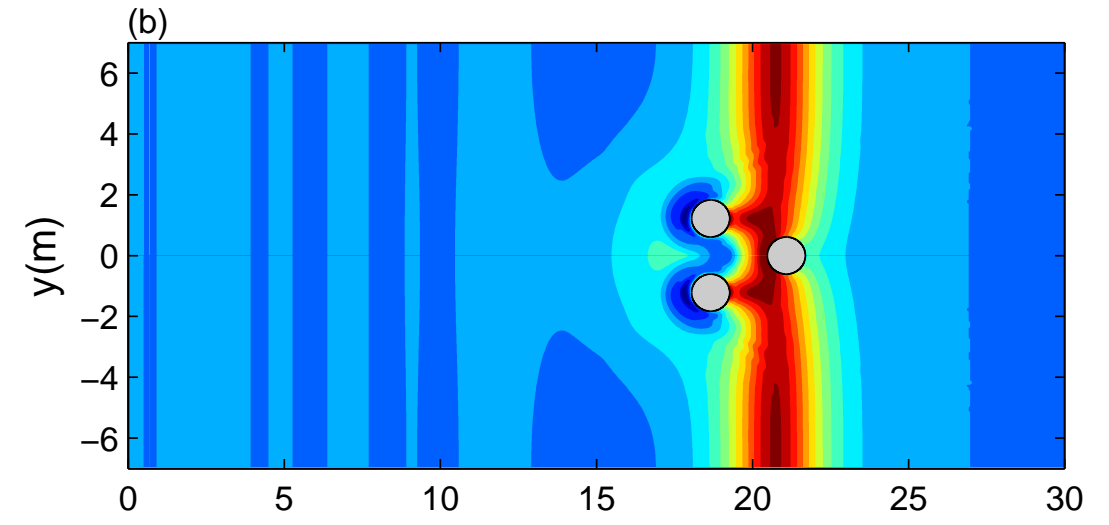

(c)

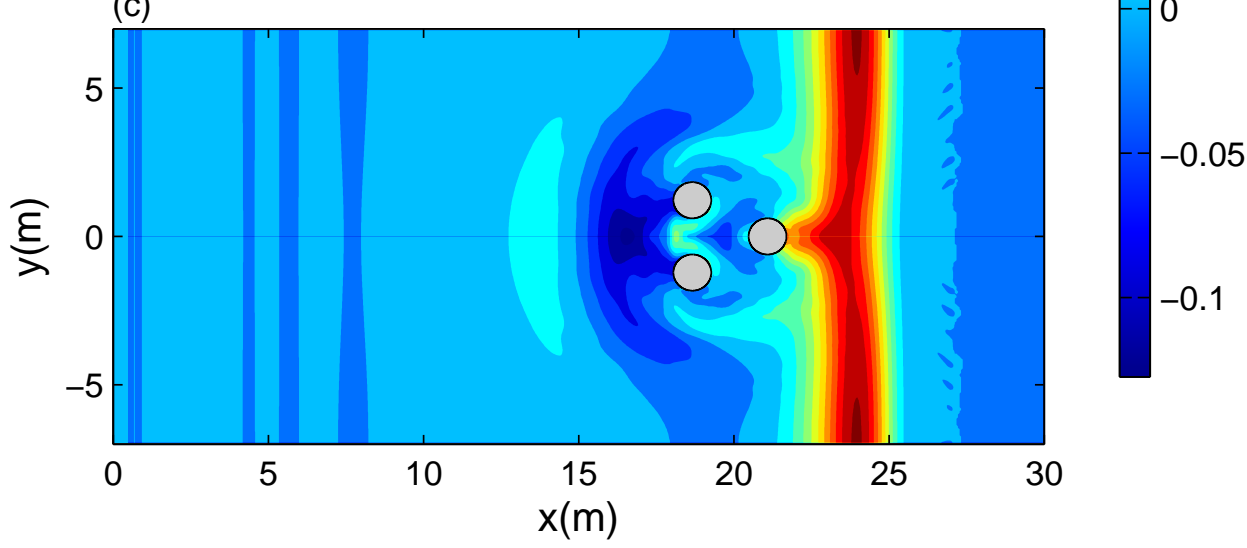



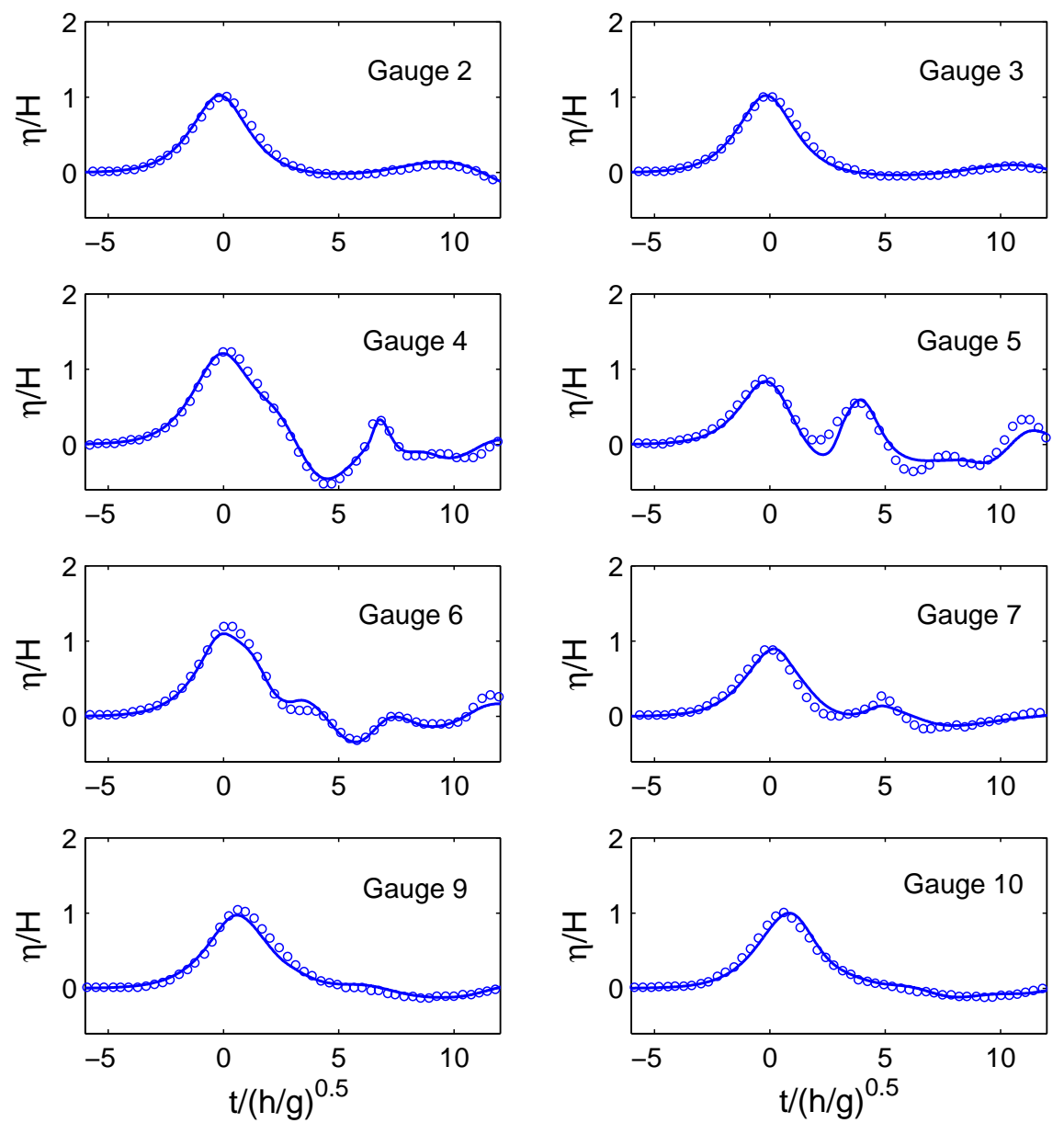

Figure 12: Model-data comparisons of temporal variations of free surface elevations at wave gauges. Circles: experimental data; solid lines: simulations. 

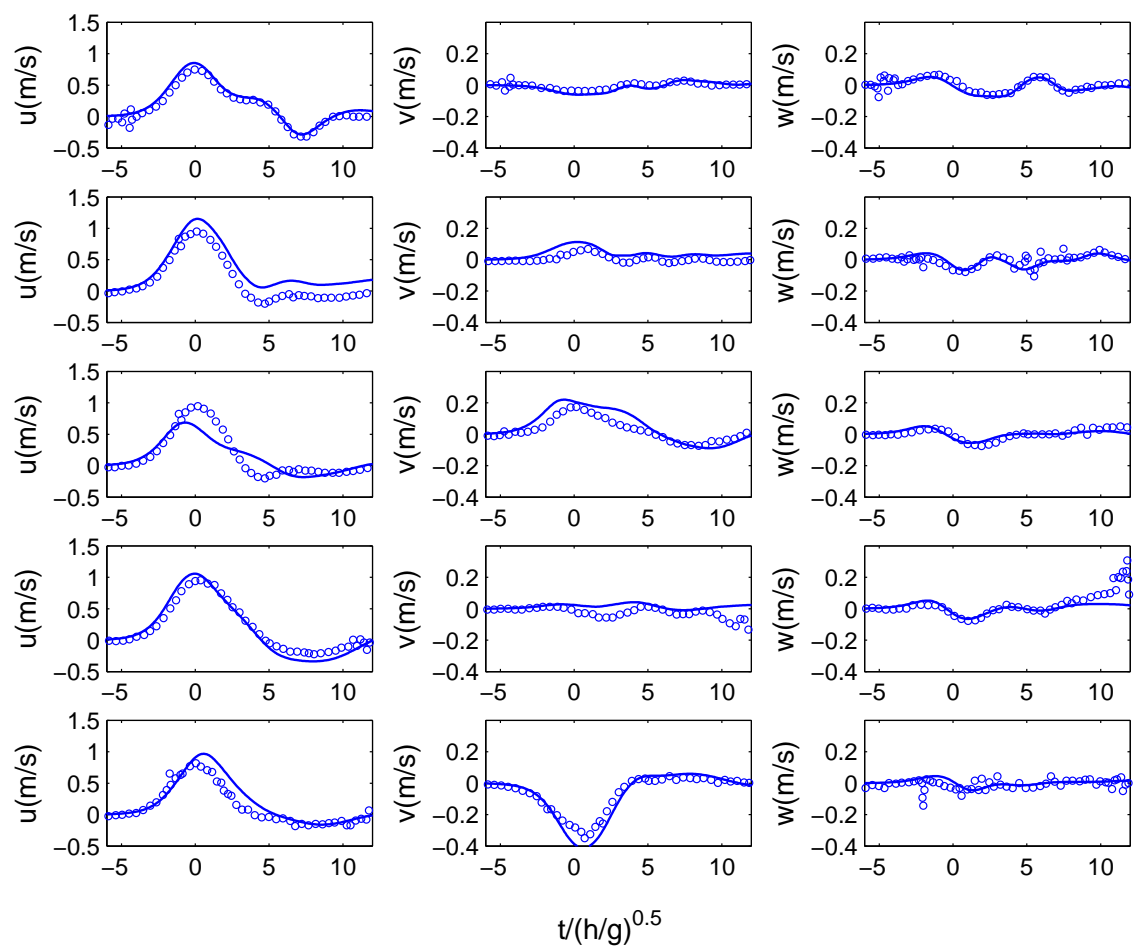

Figure 13: Model-data comparisons of temporal variations of velocities at $5 \mathrm{ADV}$ gauges. Circles: experimental data; solid lines: simulations. 

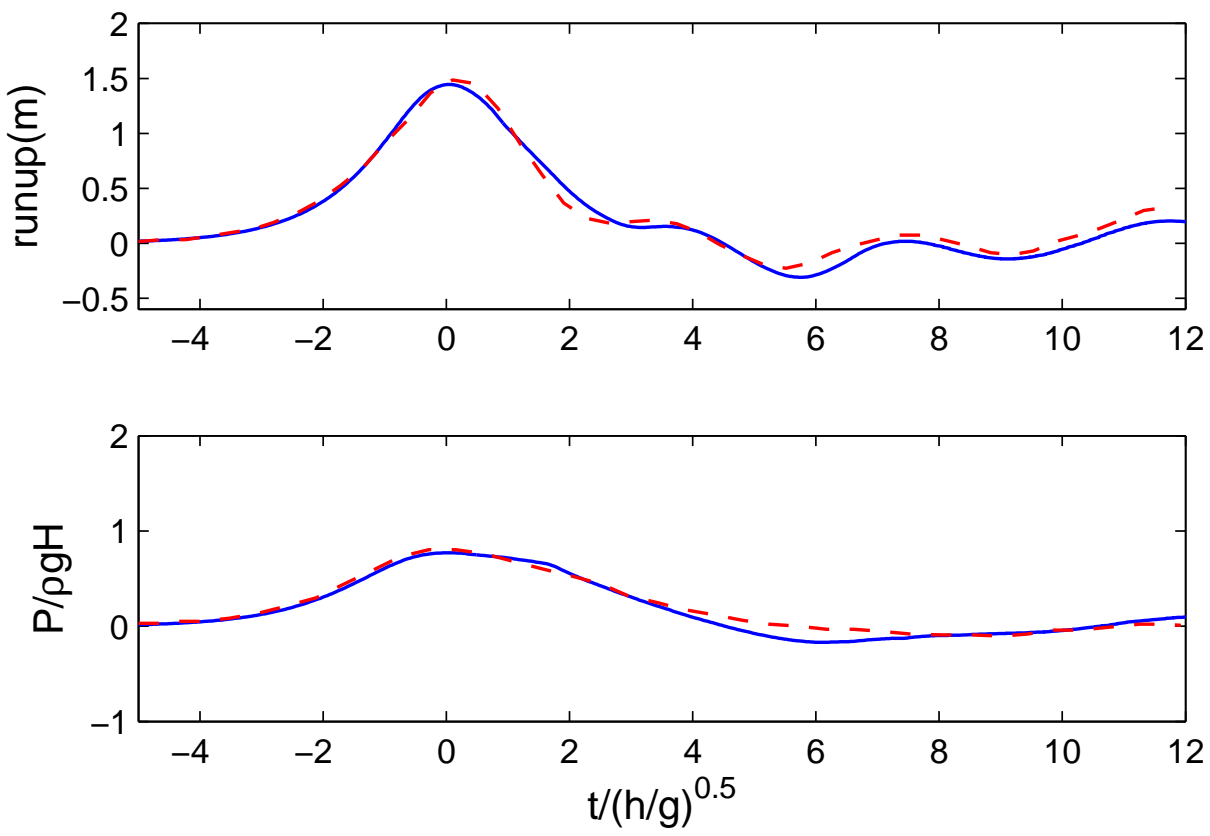

Figure 14: Model-data comparisons of wave runup and dynamic pressure in the front of the cylinder. Dashed lines: measurements; solid lines: simulations. 